\title{
Usability Assessment of a Multimodal Visual-Haptic Framework for Chemistry Education
}

\author{
Sara Comai and Davide Mazza \\ Politecnico di Milano, \\ Department of Electronics and Information (DEI), \\ Piazza L. da Vinci, 32, \\ I-20133 Milan, Italy \\ \{sara.comai, davide.mazza\}@polimi.it
}

\begin{abstract}
In this work, we assess the usability of a virtual environment where the force of interaction between the electrostatic field around the molecule and a charge associated to the proxy of a haptic device can be felt. Feedbacks to user are provided in a multimodal visual and haptic way, and auxiliary information are also rendered.
\end{abstract}

Keywords: Haptics, chemistry education, usability evaluation.

\section{Introduction}

In this work, we assess the usability of a virtual environment described in [3, 4] enabling users to probe the electrostatic field around it with a charge associated with a haptic device. The force of interaction can be felt via the haptic device, and feedbacks are provided in a multimodal way, visual and haptic.

The framework is fully described in [3]. It provides the user with a virtual environment showing a 3D representation of the molecule; different modalities of visualization are possible among the typical chemical representation of a molecule three-dimensional structure, e.g. space-filling, ball-and-stick, van der Walls surfaces, etc. Beside the graphical representation, the framework allows to feel the electronic fields' interactions by means of a haptic device (in our case, the Sensable PHANTOM (B) Omni [1]). The graphical environment shows the current position of the haptic proxy, as a yellow circle, to indicate which part of the space the user is interacting with. Auxiliary visual elements are shown to provide the user with a multimodal way of interaction, in order to integrate the information received in force, e.g., the plot of the electrostatic field along the direction connecting the proxy position and the center of the molecule, which can help in detecting maximum/minimum points of the electrostatic surface, or the force field on the molecule surface (represented by means of different colors). Figure 1 shows a screenshot of the GUI of the framework. The 3D molecule and the proxy pointer are clearly visible. The plot of the electrostatic field is shown in the upper-left corner of the picture. Further details on framework's visual and haptic capabilities can be found in [4]. 


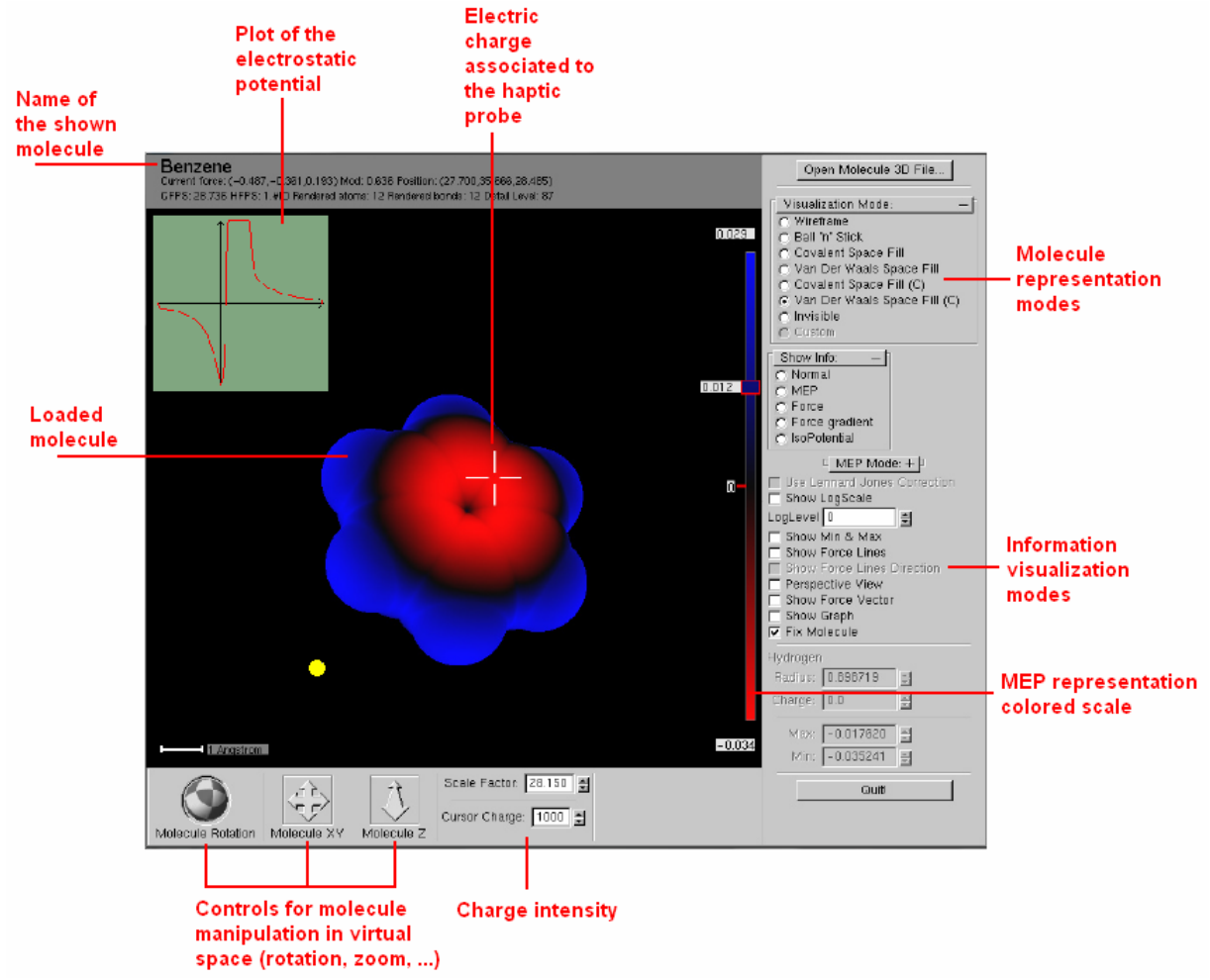

Fig. 1. A screenshot of the framework

\section{Design of the Usability Test}

Potential users of the application have been identified at first. Among the candidate users (i.e., students attending a university-level chemistry course, teachers, and chemistry researchers), being the framework mainly designed for e-learning activities, only students have been involved in this first evaluation study. The usability evaluation consisted of three stages:

- pre-questionnaire administration, sent to the users in order to collect the background information and knowledge about the topics. Beside providing some personal data on their studies and background, users were asked to rate their knowledge about both inter-molecular forces and haptic devices, on a scale ranging from 1 (I do not know at all) to 5 (I know it very well);

- tasks' execution session, where each user is asked to individually perform three tasks, consisting in identifying with the framework a set of specified electrostatic properties of a given molecule. While executing the tasks, the participants were asked to "think-out-loud". They were required to fill in a questionnaire regarding the task they are performing (the adopted questionnaire form is ASQ [5]). The following task have been performed, by following a set of instructions provided on paper: 
- Task 1 - Critical points detection: the charge is used to determine the maxima/minima of the potential in the space surrounding the molecule, giving a sensation of falling into an electrostatic surface "hole" when such points are found.

- Task 2 - Detecting polar molecules: different molecules exert a different influence on a positive or negative charge, and in different zones of the surrounding space;

- Task 3 - Feeling the anisotropy of the interaction: force effects by the molecule on the charge can change w.r.t. the charge-molecule distance and direction of approaching;

Execution of all the tasks has been supervised by us, users' behaviours observed and notes taken about what they said. The time taken by each user to complete each task has been also considered for the evaluation;

- post-questionnaire administration, providing a final evaluation of the application, by the usage of the System Usability Scale (SUS) [2]). Users were asked to answer a questionnaire to evaluate general and haptic-specific features of the application, and to finally provide an overall judgement of the experience.

A total of 5 users participated to the evaluation study of the developed chemical framework. All were students of the MSc in Computer Engineering at Politecnico di Milano. The group consisted of 2 females and 3 males with the average age of 21.6 years.

\section{Results and Discussion}

The pre-questionnaire results show that they have very little knowledge about haptic devices and an average knowledge about inter-molecular forces. With respect to chemistry softwares' background knowledge, only one participant was already using another chemistry application. The mean values of the times taken to complete each task, and the ASQ score, have been calculated. The ASQ score [5] includes the efficiency, effectiveness, satisfaction and use of haptic device usability aspects: the smaller the value, the better evaluated are these aspects by the participants. Users' feedbacks were collected regarding the different modalities of visualization and visual elements added to make haptic effects more intuitive. Based on these feedbacks, the most useful visual clues were determined as well as the problematic ones. Problems can be categorized as follows:

- $\quad$ Semiotic-problems related to the meanings of the messages of the application;

- Cognitive - cognitive effort of the user while using the application;

- Graphics - problems related to the graphical choices of the application;

- Haptic - problems related to the haptic features of the application including spatial problems and a haptic device.

Using the post-questionnaire, the score of the haptic features has been calculated using the same method as one for the SUS questionnaire [2]. The mean score of the haptic features of the application (43) is above average which shows that the 
participants found that the haptic features of the application are useful and allows user to perform the tasks in an effective and efficient way.

The SUS score of the general features of the framework is 81.5 which falls in the acceptability range of the usability of application and it is even higher than the mean score (76.2) of GUI interfaces [2] that were evaluated by SUS. The respondents rated the user-friendliness of the application in average Good, which corresponds also to the results of the SUS score.

Also the score of the final feedbacks is very high (4.3/5), confirming that the users have understood the purpose of the application, they found it attractive and would even recommend it to the colleagues.

During the test administration and as final feedbacks, students reported us the easiness by which they understood the explained concepts, thus leading to an increasing interest in the subject. Teachers also appreciated the improved awareness of the phenomena they usually explain only in theory.

\section{Conclusions and Future Work}

The framework has been tested by different users (students) on various molecules stored in a repository associated with the framework.

All the users recognized as the most important benefit the possibility of combining the typical visualization of chemical data with the rendering of the feeling of nanoscale/atomic interactions that can improve the understanding of real phenomena. As future work, given that students greatly appreciated it and they were literally enthusiastic when facing the novelty of such a tool, we plan to introduce it in teaching activities, i.e. in chemistry course as auxiliary tool to integrate classical lectures.

Acknowledgements. We would like to thank Margarita Lukjanska for her precious contribution on the usability assessment activity.

\section{References}

1. Sensable Phantom Omni. Web site, http://www.sensable.com/haptic-phantom-omni.htm

2. Bangor, J.T.M.A., Kortum, P.T.: Determining what individual SUS scores mean: Adding an adjective rating scale. Journ. of Usability Studies 4(3), 114-123 (2009)

3. Comai, S., Mazza, D.: A haptic-enhanced system for molecular sensing. In: Gross, T., Gulliksen, J., Kotzé, P., Oestreicher, L., Palanque, P., Prates, R.O., Winckler, M. (eds.) INTERACT 2009. LNCS, vol. 5727, pp. 493-496. Springer, Heidelberg (2009)

4. Comai, S., Mazza, D.: Haptic and visual rendering for multi-modal exploration of molecular information. In: Proceedings of BSC HCI (2010)

5. Lewis, J.R.: Psychometric evaluation of an after-scenario questionnaire for computer usability studies: The ASQ. SIGCHI Bulletin 23(1), 78-81 (1991) 